\title{
AD-DICE: an implementation of adaptation in the DICE model
}

\author{
Kelly C. de Bruin • Rob B. Dellink • Richard S. J. Tol
}

Received: 28 February 2007 / Accepted: 7 October 2008 / Published online: 30 January 2009

(C) The Author(s) 2009. This article is published with open access at Springerlink.com

\begin{abstract}
Integrated Assessment Models (IAMS) have helped us over the past decade to understand the interactions between the environment and the economy in the context of climate change. Although it has also long been recognized that adaptation is a powerful and necessary tool to combat the adverse effects of climate change, most IAMS have not explicitly included the option of adaptation in combating climate change. This paper adds to the IAM and climate change literature by explicitly including adaptation in an IAM, thereby making the trade-offs between adaptation and mitigation visible. Specifically, a theoretical framework is created and used to implement adaptation as a decision variable into the DICE model. We use our new AD-DICE model to derive the adaptation cost functions implicit in the DICE model. In our set-up, adaptation and mitigation decisions are separable and AD-DICE can mimic DICE when adaptation is optimal. We find that our specification of the adaptation costs is robust with respect to the mitigation policy scenarios and parameter values. Our numerical results show that adaptation is a powerful
\end{abstract}

K. C. de Bruin $(\bowtie) \cdot$ R. B. Dellink

Environmental Economics and Natural Resources Group, Wageningen University,

Hollandseweg 1, 6706KN, Wageningen, The Netherlands

e-mail: kelly.debruin@wur.nl

R. B. Dellink · R. S. J. Tol

Institute for Environmental Studies, VU University Amsterdam,

Amsterdam, The Netherlands

R. S. J. Tol

Economic and Social Research Institute, Dublin, Ireland

R. S. J. Tol

Department of Spatial Economics, VU University Amsterdam,

Amsterdam, The Netherlands

R. S. J. Tol

Department of Engineering and Public Policy, Carnegie Mellon University,

Pittsburgh, PA, USA 
option to combat climate change, as it reduces most of the potential costs of climate change in earlier periods, while mitigation does so in later periods.

\section{Introduction}

Climate change poses one of the biggest global environmental threats for current and even more so for future generations. There is a scientific consensus that human activities, through which greenhouse gases are emitted into the atmosphere, are changing our climate (IPCC 2001a). Greenhouse gases have accumulated in the atmosphere for centuries and the decay of most greenhouse gases takes more than 50 years. Therefore, the climate change problem will persist for at least the next century. Climate change is expected to do damages to the economy, with estimates of, on average, $2 \%$ of GDP (IPCC 2001b) by 2100 and perhaps much higher damages after that.

Consequently, it is important to design efficient long-term climate policies. There are two main options that a nation has in addressing climate change. Firstly it can try to limit climate change by reducing its greenhouse gas emissions or exploiting carbon sinks. This is referred to as mitigation. The other option is adaptation. Adaptation refers to adjustments in ecological, social or economic systems to moderate potential damages or to benefit from opportunities associated with climate change (Smit and Pilifosova 2001). Examples of adaptation are the building of dykes, the changing of crop types, and vaccinations.

To understand the issue of climate change, Integrated Assessment Models (IAMS) have been developed that integrate the main causes and effects of climate change. IAMs combine disciplines as to evaluate the whole cause and effect chain of climate change. In such a manner the effects of the economy on climate change and the effects of climate change on the economy are linked. The advantage of IAMS is that they can deal with important issues such as the efficient allocation of abatement burdens and accepted damages, by specifying the costs and benefits of various abatement strategies, using a detailed description of both economic and environmental developments. IAMS, therefore, can analyse what the optimal climate strategy should be. Up to now, however, IAMS focus on the trade-off between damages due to climate change and mitigation costs and ignore the option of adaptation or at best treat it implicitly as part of the damage estimate. ${ }^{1}$ Tol and Fankhauser (1998) survey the IAM literature and conclude that in the majority of the models adaptation is not included; this situation has hardly improved since then. Furthermore, mostly when adaptation was included in a model, only induced adaptation and implicit adaptation were considered. Induced adaptation refers to the process of readjustment to the new climate and it represented through transition costs and transition time. Implicit adaptation refers to where adaptation is considered within a relationship in the model using certain assumptions. This is often in the form of the damage function assuming optimal adaptation or no adaptation. Adaptation is then not a variable that can be changed or studied, i.e. it is not explicit. Explicit adaptation refers to adaptation

${ }^{1}$ Examples of such models are: MERGE (Manne et al. 1995, 2005), FUND (Tol 2006) and IGSM (Sokolov et al. 2005). 
being modelled as an endogenous choice variable that can be set at a specific level or optimised. Including adaptation explicitly gives you the opportunity to study the exact levels, costs, and effects of adaptation.

Because of the private nature of most adaptation, it is sometimes argued that adaptation is not a decision variable for a region, as the adaptation decisions are not in the hands of the leaders of that region (Tol 2005). However, besides the fact that many forms of adaptation are public, private adaptation is still a decision taken in a region, even if not by the leaders of that region. One may also argue that, under certain assumptions, the socially optimal adaptation (as calculated below) coincides with the adaptation provided by the market. This, however, is unlikely as the public lacks information on the effects of climate change and adaptation options, and adaptation often entails big scale projects that the market cannot provide. Studies have shown that the effects of adaptation can be enormous, decreasing gross damages by up to $80 \%$ (Mendelsohn 2000). Therefore, to fully understand the effects of climate change and climate change policies adaptation also needs to be considered and modelled as a policy variable.

In this paper, we include adaptation as a policy variable in an IAM, namely the Dynamic Integrated model of Climate and the Economy (DICE), which was originally developed by Nordhaus (1994) and elaborated in Nordhaus and Boyer (2000). The DICE model is a global model and includes economic growth functions as well as geophysical functions. The use of adaptation is assumed to be optimal and is already included in the damage function. We develop what we have named the Adaptation in DICE (AD-DICE) model that includes adaptation as a decision variable.

Estimates from empirical literature on the costs and benefits of adaptation are used to calibrate the model and derive the adaptation cost curve that is implicit in the DICE model. We then construct new policy scenarios with no adaptation to understand the effects of adaptation. We take a closer look at the effects of adaptation and mitigation on each other.

This paper is structured as follows; in the next section the literature on adaptation in IAMs is reviewed. Section 3 lays out the AD-DICE model and its theoretical framework. In Section 4 the details of the calibration are described and the parameter values of the AD-DICE model are given. Section 5 presents the results with an analysis of the different policy options and the interactions of mitigation and adaptation. In Section 6 a sensitivity analysis is done. Finally we conclude and make a few suggestions for further research.

\section{Literature review}

There have been many articles calling for more research on adaptation, especially in the modelling context. Tol and Fankhauser (1998) specifically call for the introduction of explicit adaptation in IAMS. They survey 20 IAMS and find that adaptation is distinguished only in very few of the models. Furthermore, they find only one model, the Policy Analysis of the Greenhouse Effect (PAGE) model (Hope et al. 1993; Plambeck et al. 1997), where adaptation is considered as a policy variable for all impact sectors. Since Tol and Fankhauser (1998), nothing has really changed with regard to the inclusion of adaptation in IAMS. This is confirmed by a recent review of various sorts of adaptation models by Dickinson (2007). Some important theoretical work has been developed simultaneously to our analysis (Lecocq and Shalizi 2007). 
Hope et al. (1993) investigate two adaptation policy choices in the PAGE model, namely no adaptation and aggressive adaptation. Aggressive adaptation decreases initial climate change impacts by up to $90 \%$. Hope et al. (1993) say that this reduction will cost 0.5 trillion Euros and decrease impacts by 17.5 trillion Euros. These estimates do not seem realistic and contradict existing empirical literature on the costs and effects of adaptation (Reilly et al. 1994; Parry et al. 1998a, b; Fankhauser 1998; Mendelsohn 2000). The benefits of adaptation estimated by Hope et al. (1993) are much higher than is found in this literature. Not surprisingly, Hope et al. (1993) find that an aggressive adaptation policy is beneficial and should be implemented. It should be noted that the authors of this article also call for incorporating improved information on adaptation in their model. Hope et al. (1993) model adaptation such that it decreases the "tolerable" rate of climate change, the "tolerable" absolute climate change and the impacts beyond these "tolerable" amounts. Although Hope et al. (1993) take a step in considering adaptation and how it may be implemented into IAMS; the values used are very unrealistic and teach us little about dynamics of adaptation or the trade-offs with mitigation. Furthermore, adaptation is not a continuous choice, but a discrete variable; and it is a scenario variable rather than a choice variable. Later versions of the PAGE model have been developed, however, the same specification of adaptation were used (Hope 2006).

A more recent and detailed paper where adaptation is explicitly modelled is that of Tol (2007). This model only considers sea level rise and coastal protection. It models protection as a continuous decision variable, based on Fankhauser (1994), and gives insights into adaptation dynamics. Tol (2007) shows that protection is a very important option to combat the impacts of sea level rise. Furthermore mitigation and adaptation need to be traded off as more mitigation will lead to less free resources for adaptation. Tol and Dowlatabadi (2001) did a similar analysis looking at vectorborne diseases, focusing on malaria. They find that overly ambitious mitigation could increase the adverse impacts of climate change due a decrease in adaptive capacity.

Previous papers have taken the first steps to explicitly including adaptation in IAMS, but clearly do not exhaust the potential. Adaptation is an important policy option that needs to be included in IAM analyses for all climate change damages. This article adds to the IAM and climate change literature by doing that.

\section{A framework with explicit adaptation decisions}

In the DICE model, utility is maximized. Utility is calculated as the discounted natural logarithm of consumption. In each time period, consumption and savings/ investment are endogenously chosen subject to available income reduced by the costs of climate change (residual damages, mitigation costs and protection costs). The climate change damages are represented by a damage function that depends on the temperature increase compared to 1900 levels.

Adaptation to climate change would decrease the initial damages of climate change. This is the mechanism we add to the DICE model. We define gross damages as the initial damages by climate change if no changes were to be made in ecological, social and economic systems. If these systems were to change to limit climate change damages (i.e. adapt) the damages would decrease. We refer to these "left-over" damages as residual damages. Reducing gross damages, however, comes at a cost, 
i.e. we need to invest resources in adaptation. These costs are referred to as protection costs or adaptation costs. Thus, the net damages in DICE are the total of the residual damages and the protection costs.

In the DICE model the net damage function is a combination of the optimal mix of protection costs and residual damages. ${ }^{2}$ Thus the net damage function given in DICE can be unravelled into residual damages and protection costs. We do this as follows. The original damage function in DICE is given as

$$
\frac{D_{t}}{Y_{t}}=a_{1} \mathrm{TE}_{t}+a_{2} \mathrm{TE}_{t}^{2},
$$

where $D_{t}$ represents the net damages, $Y_{t}$ the output and $\mathrm{TE}_{t}$ the temperature change compared to the 1900 temperature. We split the net damages into residual damages $\left(\mathrm{RD}_{t}\right)$ and protection costs $\left(\mathrm{PC}_{t}\right)$.

$$
\frac{D_{t}}{Y_{t}}=\frac{\mathrm{RD}_{t}\left(\mathrm{GD}_{t}, P_{t}\right)}{Y_{t}}+\frac{\mathrm{PC}_{t}\left(P_{t}\right)}{Y_{t}}
$$

Here we assume that the protection costs and the residual damages are separable, and can be represented as a fraction of income. They both depend on protection $\left(P_{t}\right)$ but the costs are independent of each other. Residual damages depend on both the gross damages $\left(\mathrm{GD}_{t}\right)$ and the level of protection $\left(P_{t}\right)$. Effectively, this makes the decisions on the levels of protection and mitigation separable.

We assume that the gross damage function takes the form given in:

$$
\frac{\mathrm{GD}_{t}}{Y_{t}}=\alpha_{1} \mathrm{TE}_{t}+\alpha_{2} \mathrm{TE}_{t}^{\alpha_{3}}, \quad \text { where } \quad \alpha_{2}>0 \text { and } \alpha_{3}>1
$$

This is the most commonly used form for damage costs of climate change in IAMS, where $\alpha_{3}$ generally takes a value between 1 and 3 (Tol and Fankhauser 1998). This is also the same form as used in the DICE model, however, $\alpha_{3}$ is not assumed to be 2 as in the DICE model, but is left to be determined through calibration.

We use the following function to express $\mathrm{RD}_{t}$ as a function of $P_{t}$ and $\mathrm{GD}_{t} ;^{3}$

$$
\mathrm{RD}_{t}=\mathrm{GD}_{t} \times\left(1-P_{t}\right), \quad \text { where } 0 \leq P_{t} \leq 1 .
$$

The main advantage of using the form given in Eq. 4 is that $P$ has an intuitive interpretation. Protection is then given on a scale from 0 to 1 , where 0 represents no protection: none of the gross climate change damages are decreased through protection. A value of 1 would mean that all gross climate change damages are avoided through adaptation. Thus, $P$ gives the fraction by which gross damages are reduced: $P_{t}=\frac{\mathrm{GD}_{t}-\mathrm{RD}_{t}}{\mathrm{GD}_{t}}$.

Protection costs are given as a function of the level of protection. We assume that this function is growing at an increasing rate, as cheaper and more effective

\footnotetext{
${ }^{2}$ According to Nordhaus and Boyer (2000), adaptation is included in the damage estimates and it is implicitly assumed that this is the optimal adaptation.

${ }^{3}$ In line with DICE99 online version used in this analysis, we assume that net climate change damages are strictly non-negative, that is climate change will have a negative effect. Note that this may not be the case for several regions of the world (e.g. Tol 2002) and in other models such as the DICE99 Nordhaus and Boyer (2000) version.
} 
adaptation options will be applied first, and more expensive and less effective options will be used after these, thus

$$
\frac{\partial \mathrm{PC}_{t}}{\partial P_{t}}>0 \text { and } \frac{\partial^{2} \mathrm{PC}_{t}}{\partial P_{t}^{2}}>0 .
$$

There are many types of functions that fit these criteria. We assume this function takes the form of a power function ${ }^{4}$

$$
\frac{\mathrm{PC}_{t}}{Y_{t}}=\gamma_{1} P_{t}^{\gamma_{2}}, \quad \text { where } \quad \gamma_{1}>0 \text { and } \quad \gamma_{2}>1
$$

We assume that the level of protection is chosen every time period (10 years). The protection in one time period does not affect damages in the next period, thus each decade the same problem is faced, and the same trade-off holds. This implies that both the costs and benefits of adaptation are "instantaneous", i.e. they fall within the same time period, that is within the same decade. The important implication of this assumption is that as long as adaptation is applied optimally, the benefits of adaptation will always outweigh the costs (this follows directly from the maximisation) and hence the adaptation decision will never draw away funds from mitigation policy. Although this way of modelling adaptation benefits and costs is debatable, many adaptation measures have this characteristic. Examples of such adaptation measures are applying sunblock, switching on air conditioning, changing holiday destinations and changing crop types. Other adaptation measures, especially in the category of anticipatory adaptation, however, have a time-lag in costs and benefits. Example of such measures are building seawalls and early warning systems. An analysis which adds an adaptation capital stock to represent anticipatory adaptation would, however, be interesting and is deferred to future work.

Equation 5 gives us an adaptation cost curve. Combined with Eq. 4, it compares the reduced damages (as a fraction of gross damages) with the costs of the adaptation (as a fraction of output).

\section{Calibration of the AD-DICE model}

In our analysis we use the GAMS version of the DICE99 model as available online. ${ }^{5}$ It should be noted that a new version of this model DICE2007 has recently become available. The newer version assumes decreasing abatement costs as opposed to increasing assumed in DICE99. Furthermore the damages function is higher in the newer version. de Bruin et al. (forthcoming) show that these improvements do not essentially change our results, although they increase the importance of mitigation compared to adaptation somewhat. We calibrate the AD-DICE model using the optimal control scenario of DICE. We calibrate the model in such a way that it best replicates the results of the original DICE model. To do this we constructed a model that minimized the discounted squared difference between net damages $\left(D_{t}\right)$ in the

\footnotetext{
${ }^{4}$ In the Appendix 1 another representation is presented and used to calibrate the AD-DICE model.

${ }^{5}$ This model was available on the homepage of William Nordhaus until spring 2006.
} 
Table 1 Parameter values from AD-DICE in the optimal scenario calibration

\begin{tabular}{lllllll}
\hline Parameter & $\alpha_{1}$ & $\alpha_{2}$ & $\alpha_{3}$ & $\gamma_{1}$ & $\gamma_{2}$ & $p$-value \\
\hline Value & 0.0012 & 0.0023 & 2.32 & 0.115 & 3.60 & 0.99 \\
\hline
\end{tabular}

original DICE and net damages $\left(\mathrm{RD}_{t}+\mathrm{PC}_{t}\right)$ in $\mathrm{AD}$-DICE, holding $\mathrm{TE}_{t}$ at the level obtained in the DICE optimal control scenario.

We use additional information for the calibration of the parameters: $\alpha_{1}, \alpha_{2}, \alpha_{3}$, $\gamma_{1}$ and $\gamma_{2}$. Firstly, the $P_{t}$ chosen must be optimal. Thus for all $P_{t}, \frac{\partial D_{t}}{\partial P_{t}}=0$ must hold, or $\frac{\partial \mathrm{RD}}{\partial P}=\frac{\partial \mathrm{PC}}{\partial P}$. Next, we have to identify a point on the protection cost curve to be able to calibrate the function. We chose to calibrate on the same point as used to calibrate the damages in the original DICE model: a doubling of $\mathrm{CO}_{2}$ concentrations. This is assumed to occur after 130 years $(t=13)$, this corresponds in the DICE model to a temperature rise of $2.3^{\circ} \mathrm{C}(\mathrm{TE}=2.3)$ compared to 1,900 . The second condition states that PC takes a value of $7-25 \%$ of total damages in the calibration point. This condition is taken from an estimate by Tol et al. (1998), which is based on an extensive review of impact assessment literature. We also restrict the parameter $P$ based on literature (Parry et al. 1998a; Reilly et al. 1994; Fankhauser 1998; Mendelsohn 2000) at a level between 0.3 and 0.8 at the calibration point. Furthermore, according to Tol et al. (1998), PC should lie between $0.1 \%$ and $0.5 \%$ of GDP at the calibration point; this restriction was also implemented.

Our AD-DICE model, when calibrated with these restrictions, is able to reproduce the damages of the DICE model well. To test the significance of our models we regress the DICE damages on those of the AD-DICE and test the hypothesis that they are equal using an $F$-test. The $p$-value, which may be interpreted as the chance that they are indeed equal, is given in Table 1, along with the parameter estimates.

The very high $p$-value indicates that DICE and AD-DICE are almost identical. Figure 1 depicts the net climate change costs estimated by AD-DICE and those
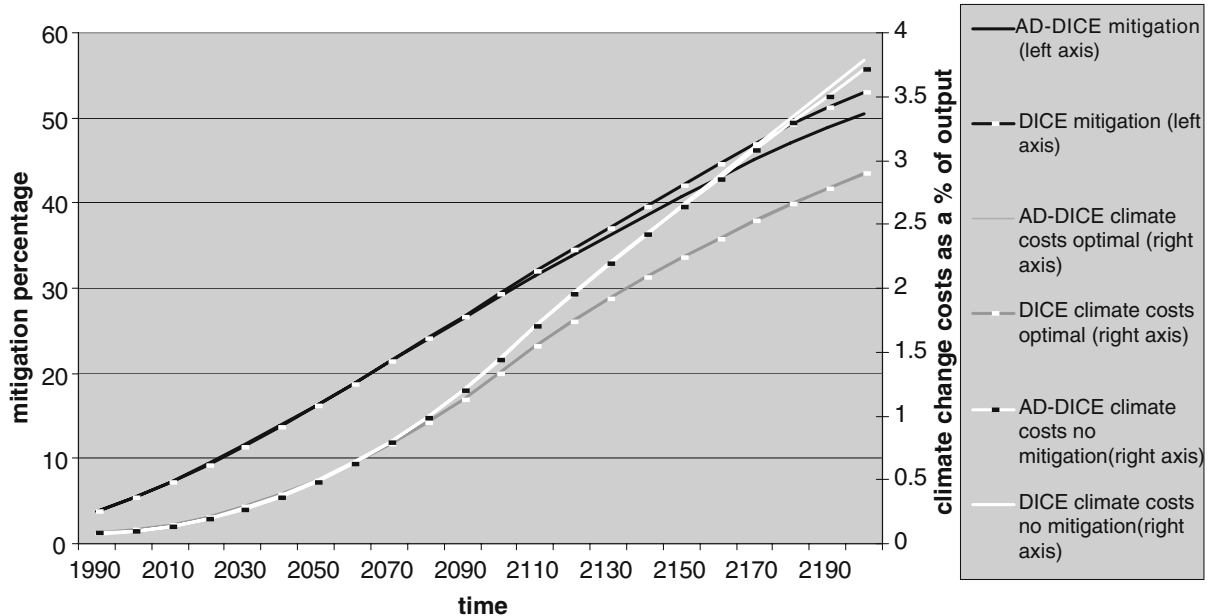

Fig. 1 Net climate change costs and mitigation levels estimated by AD-DICE and DICE: optimal scenario, no mitigation scenario 


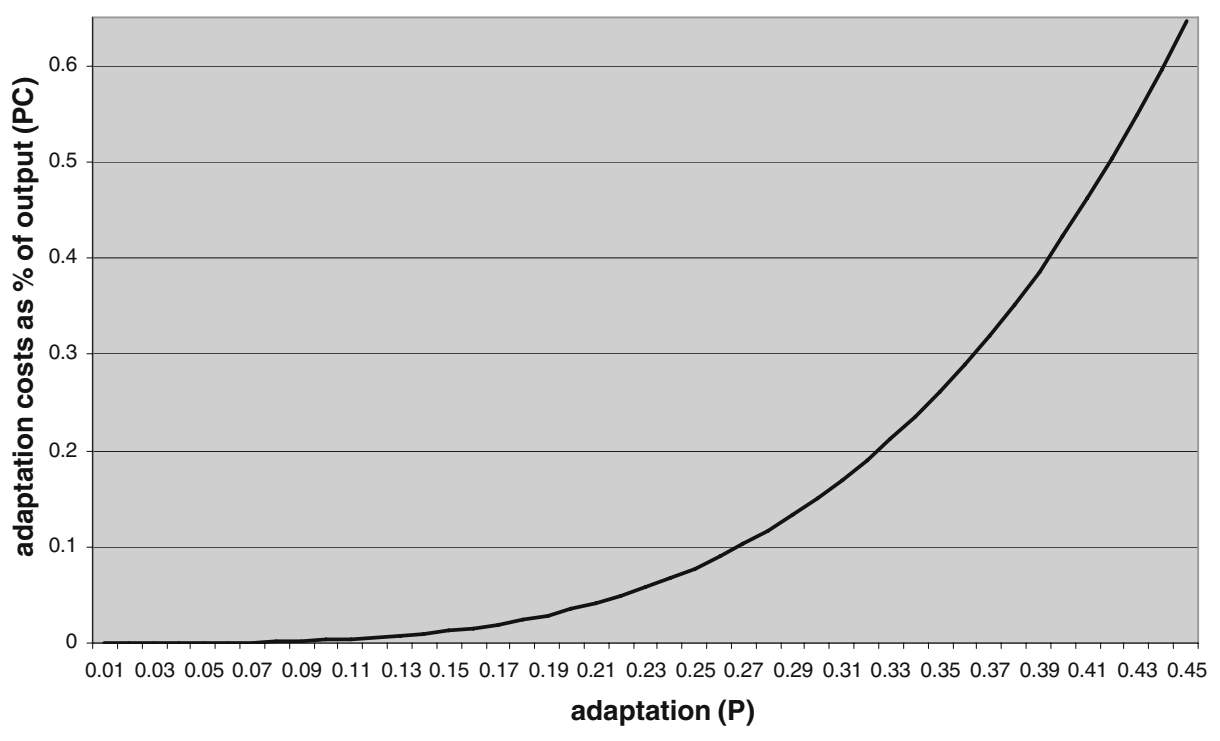

Fig. 2 The adaptation cost curve implicit in the DICE model (range 0-0.45)

estimated by DICE, for both the optimal control scenario and for the scenario without mitigation. For both scenarios, our calibration fits well with some small diversions in later periods (the present value of these differences are negligible due to the positive discount rate used). This shows that our parameter values are valid for both scenarios.

As also can be seen in Fig. 1, the AD-DICE levels of mitigation replicate those of DICE, albeit that the levels are slightly lower in later periods in AD-DICE. Mitigation is essentially the same in DICE and AD-DICE. This is because the decisions to mitigate and adapt are separable. In DICE, mitigation is set by the marginal damage cost. In AD-DICE, mitigation is set by the marginal residual damage plus adaptation cost. As the net damage profiles in DICE and AD-DICE are practically identical, so are the marginals. Thus our model will give the same results as the DICE model if we keep adaptation at its optimal level.

Using the parameter values found in AD-DICE we can draw an adaptation cost curve, given in Fig. 2. We see that the protection costs of the first $15 \%$ of gross damage reduction are extremely low after which they rise. The optimal level of adaptation varies from 0.09 to 0.45 , with an average of 0.33 , that is $33 \%$ of gross damages are reduced due to adaptation. It can easily be seen that the costs of adaptation rise to such a high level that it can never be optimal to fully adapt to climate change. That is solely adapting to climate change is not a solution and mitigation will be needed too.

\section{Results}

In this section we present our results. We first look at how climate change costs are composed. Then we compare different policy options. Finally we look at the dynamics of adaptation and mitigation and how they affect each other. 
5.1 Costs of climate change and policy options

The costs of climate change include residual damages, protection costs and abatement costs. In Fig. 3, we see that a large part of climate change costs consist of residual damages in the optimal control scenario. Mitigation costs increase relatively steeply over time.

Now we compare different policy options using the AD-DICE model. We take two climate change policy scenarios created by Nordhaus and Boyer (2000) as a starting point. We revise these scenarios by including non-optimal adaptation. We then compare the effects of the different scenarios on the basis of utility.

The two scenarios of Nordhaus and Boyer (2000) are the no control baseline scenario and the optimal control scenario. In the no control scenario, adaptation is chosen at its optimal level, thus in such a way as to maximise utility, but the level of mitigation is set at zero. In the optimal control scenario both adaptation and mitigation are chosen at their optimal levels. We use four scenarios in AD-DICE: optimal control, no-control, only-adaptation and only-mitigation. Our optimal case is the same as that of Nordhaus and Boyer (2000). In our no-control scenario, adaptation and mitigation levels are set at zero. In the only-adaptation scenario, the adaptation is at its optimal level while the mitigation level is zero. In the only-mitigation scenario, the mitigation is at its optimal level while the adaptation level is zero.

We first look at the utility of the different scenarios. Logically we see that the optimal control leads to the highest welfare level, followed by the only adaptation scenario. This is followed by the only-mitigation scenario. Finally the scenario that creates the lowest utility is the no-control scenario. Thus only adapting results in

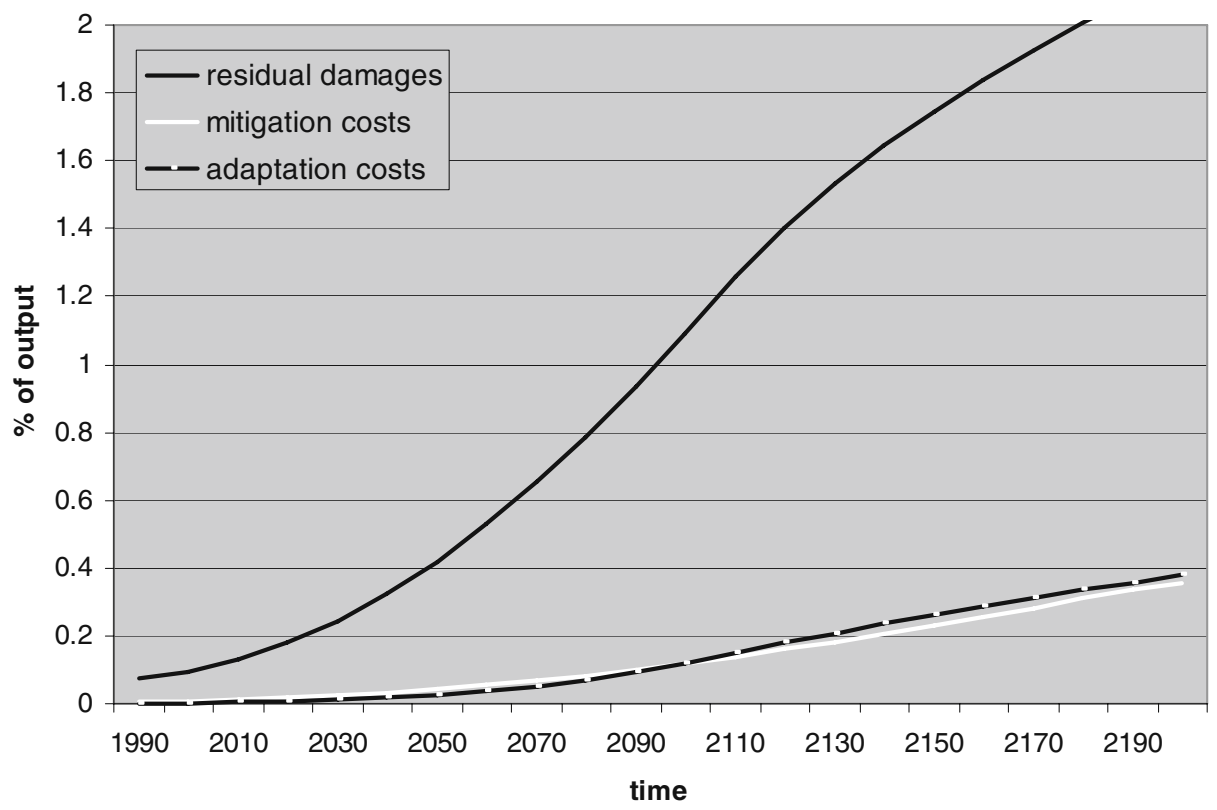

Fig. 3 Composition of climate change costs in the optimal scenario 


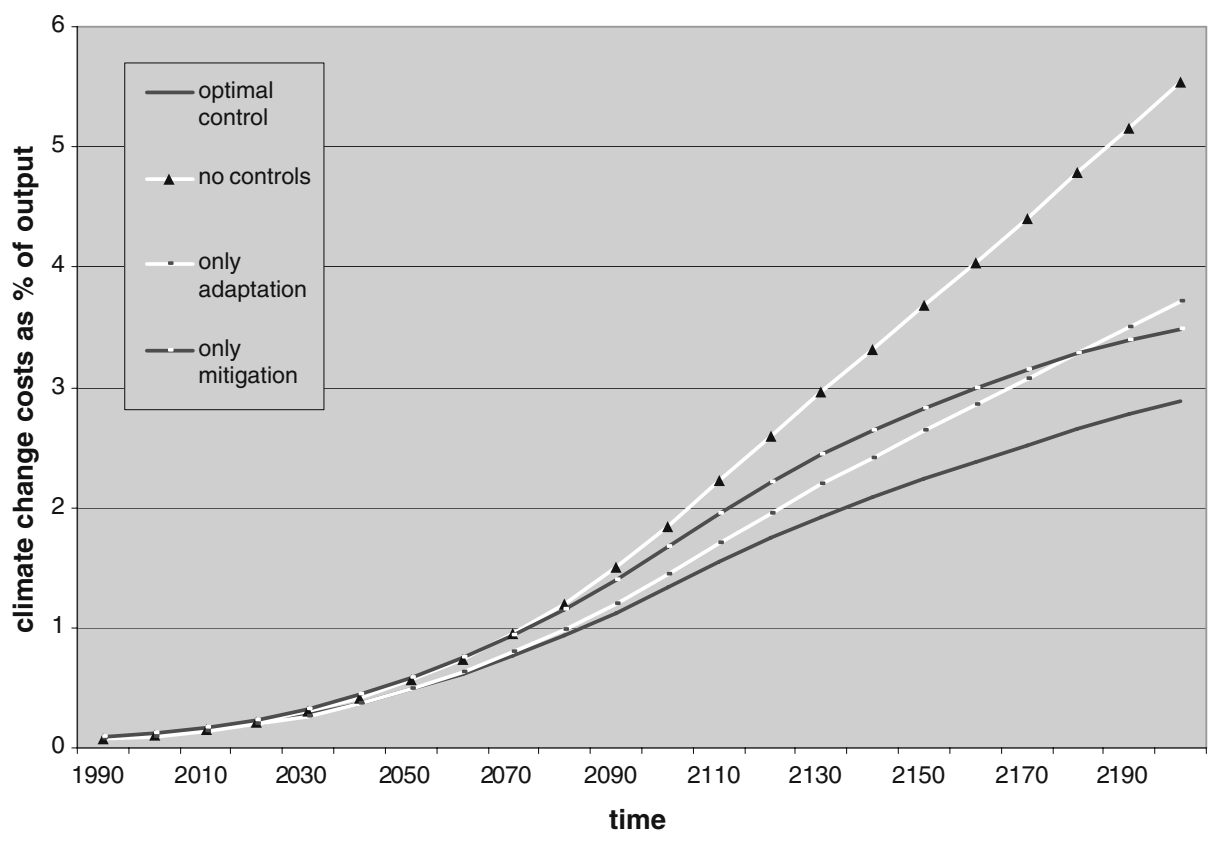

Fig. 4 Climate change costs of different policy scenarios

a higher utility than only mitigating and adaptation is the preferred, single control in this model. These results confirm the importance of adaptation. Note that this conclusion may be specific to our model setting. Below, we report the results of a sensitivity analysis that show that our conclusions are robust to a range of parameter variations. More fundamental changes in model assumptions, however, may result in a different conclusion.

In Fig. 4, we compare the distribution of costs of climate change over time with the different policy scenarios. The different scenarios distribute the cost of climate change quite differently over time. Thus changes in the discount rate will also change the ranking of the scenarios.

We see that in the only-mitigation scenario damages in the last periods are much smaller than in the no-control scenario. The only-adaptation scenario damages are smaller in the beginning periods compared to the no-control scenario. The optimal scenario distributes the damages more evenly over time. At certain points in time it is less beneficial than a scenario with only adaptation, but overall this scenario has the least discounted damages.

\subsection{Mitigation and adaptation}

There is increasing attention for the interactions between adaptation and mitigation (Klein et al. 2007). Extra investment in adaptation may decrease or increase emissions, or mitigation may stimulate or hinder adaptation. An example of this is increased use of air conditioning in the face of global warming, which increases 
emissions. This relation is surveyed in AR4 (Klein et al. 2007), they conclude that the relationship between adaptation and mitigation is ambiguous and small. Here we assume that adaptation and mitigation have no direct effects on each other.

Adaptation and mitigation are rival goods, as they compete for resources. Increased mitigation (adaptation) will decrease the funds available for adaptation (mitigation). This is explicitly included in our set-up, but is not particularly important as the sum of money spent on either adaptation or mitigation is small relative to the total budget. Furthermore, in the situation of optimal adaptation the immediate benefits will always be higher than the costs and will therefore not deplete funds for mitigation in this set-up. As mentioned in the model description, our model specification ignores the sometimes considerable time-lags between the costs and benefits of adaptation measures, and assumes that benefits will occur in the same decade as the associated costs.

More importantly, we model adaptation and mitigation as policy substitutes. The word substitute is used here in the economic sense of the word: more mitigation (adaptation) reduces the need for adaptation (mitigation). In the optimal policy, however, there is both adaptation and mitigation, as mitigation cannot avoid all climate change, and adaptation cannot avoid all impacts.

To examine the dynamics of adaptation and mitigation in more detail, we look at the net benefits of adaptation and mitigation over time (both as a percentage of output) in Fig. 5. We see that adaptation has higher benefits until 2140 than does mitigation. After that, however, mitigation becomes much more beneficial than adaptation. Thus even though it is optimal to invest in mitigation, few of these benefits will be felt in the first 60 years-largely because of the slow workings of the carbon cycle and climate.

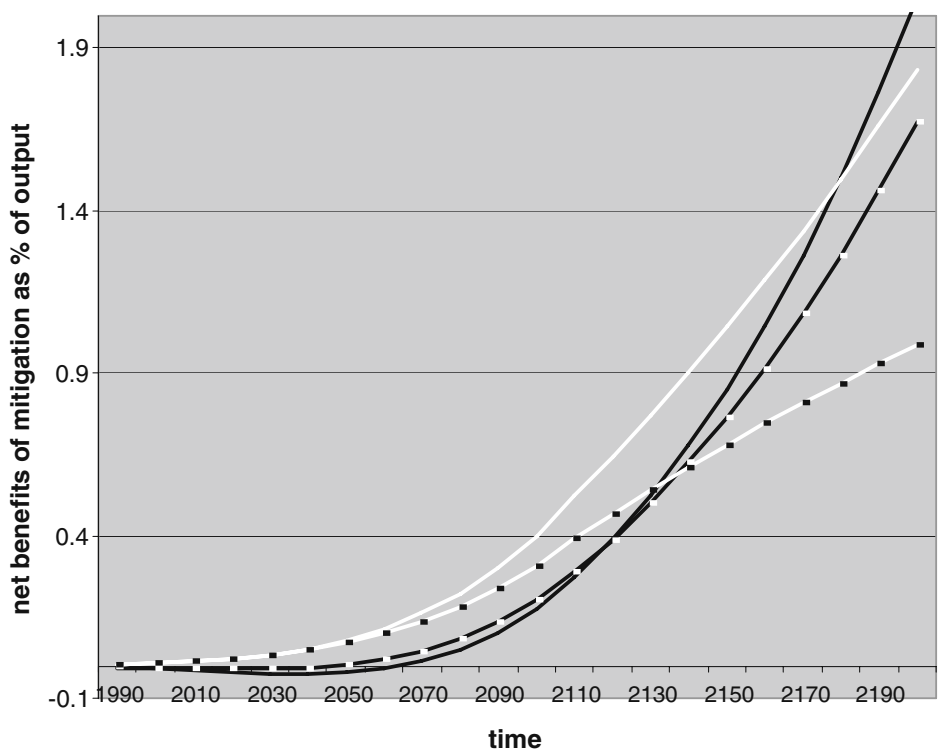

Fig. 5 Net benefits of adaptation and mitigation over time and scenarios

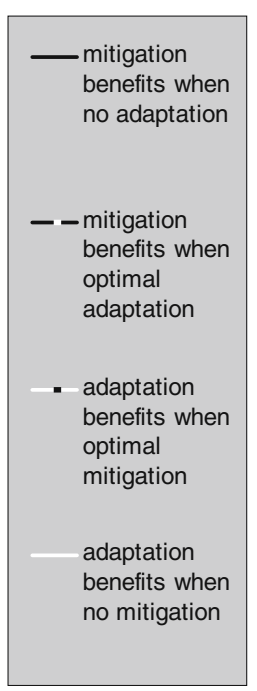

\footnotetext{
$$
\text { . }
$$$$
\text { . }
$$ 
Furthermore, we look at how adaptation and mitigation affect each other. In Fig. 5, the net benefits of mitigation are given for the case when only mitigation is an option and for the case where adaptation is also possible. We see that including adaptation as an option increases the benefits of the optimal path of mitigation until 2120 but decrease the benefits later. This is because adding adaptation as a control option will decrease the optimal level of mitigation. Because of this, costs of mitigation in the beginning periods will be avoided but this also entails that benefits of mitigation in later periods are lost. In this case, over-investment in mitigation in earlier periods is unnecessary because the option of adaptation is available. That is, adaptation is a more effective short-term tool, which can be applied instead of the less effective long-term tool of mitigation. In later periods, adaptation decreases the effectiveness of mitigation in reducing impacts. As more is invested in adaptation, the less effective mitigation will become. The other way around more adaptation decreases residual damages compared to gross damages making mitigation less effective.

There is a chance that policymakers do not look at the optimal control over time but only consider the near future that is the world has a myopic view. In this case because the benefits of adaptation are higher in earlier years, there may be an overinvestment in adaptation and an underinvestment in mitigation. This is a problem of inter generational externalities, that is the burdens of mitigation are felt in earlier periods, while the benefits are reaped in later periods (or more accurately the benefits of a high level of consumption are reaped now, while the burdens are felt by later generations in the form of climate change damages). However, a side benefit in this respect is that the net damages of the optimal level of mitigation in the earlier periods are lower and even become benefits when adaptation is included as a control option.

We see that mitigation only has a "negative" effect on adaptation. In Fig. 5 we see that adaptation is less beneficial when mitigation is also an option. This is because mitigation reduces gross damages reducing the potential benefits of adaptation. The difference between benefits of adaptation with optimal mitigation and without mitigation increases over time, because the effect of mitigation becomes more pronounced over time. It is important to note that even though mitigation and adaptation decrease each others effectiveness, i.e. there is a negative interaction effect; the simultaneous use of both options is still optimal. The marginal costs of both options increase more than proportionately with higher percentages of mitigation/adaptation, and this effect is stronger than the interaction effect, and the optimal policy involves a mixture of moderate amounts of both adaptation and mitigation.

\section{Sensitivity analysis}

In this section we conduct a sensitivity analysis. We have already seen that our model is robust to changes in the scenario used in the calibration, see Fig. 1. AD-DICE replicates DICE in all scenarios where adaptation is optimal and does not need to be recalibrated for different scenarios. Next we check if our model is robust to changes in the climate sensitivity and the discount rate used. 


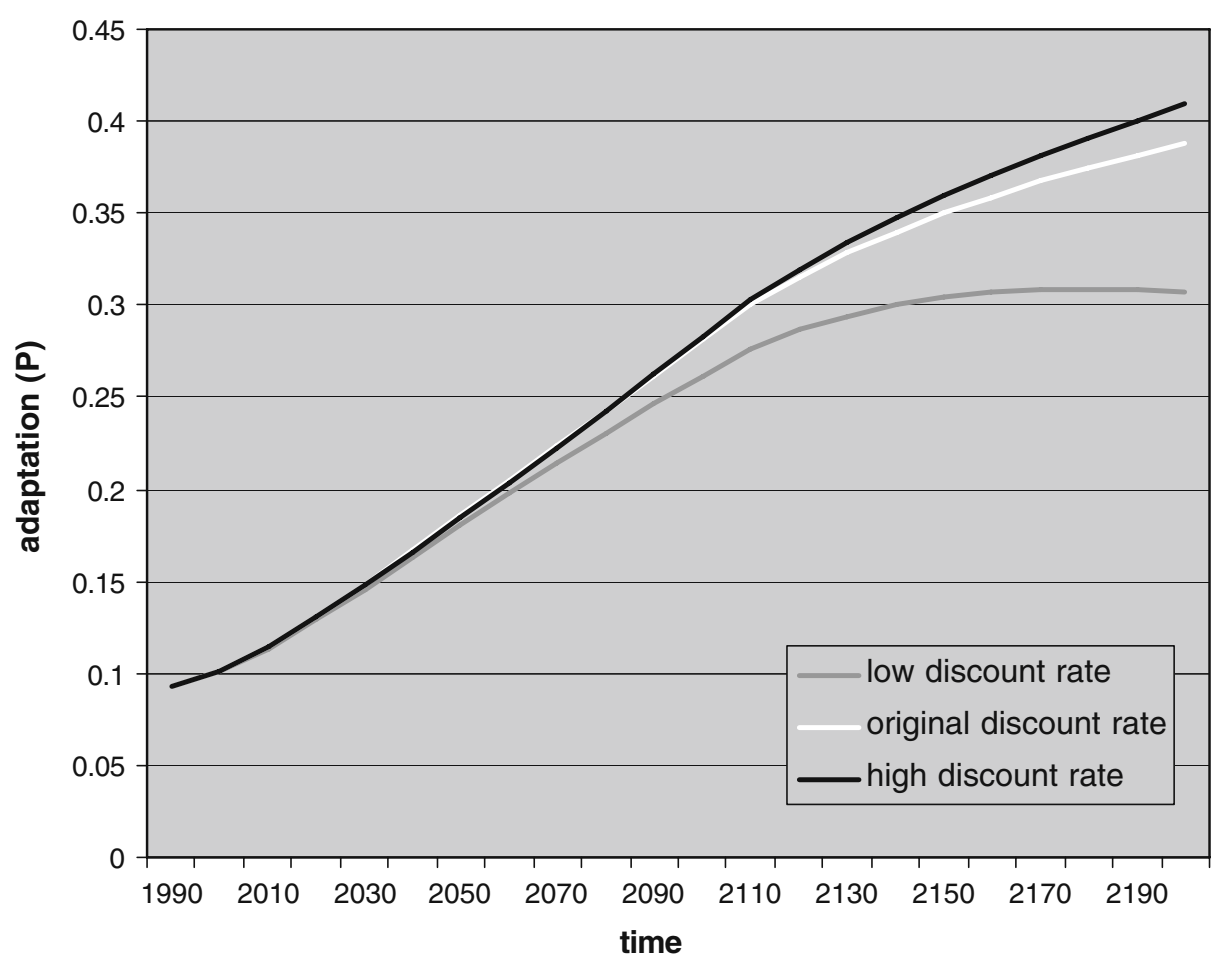

Fig. 6 Adaptation level with different discount rates

\subsection{Discount rate}

We run both the DICE and AD-DICE model with three alternative utility discount rates, namely a low level, an original, middle level and a high level. The original discount rate is 0.03 and decreases over time, the low level discount rate is $50 \%$ of the original level (0.015) and the high level is $150 \%$ of the original level (0.045). We see that the AD-DICE model adjusts to changes in the discount rate in the same way as the DICE for all relevant variables. We find that the AD-DICE model has slightly lower levels of mitigation but replicates the DICE model very well. Thus without recalibration the model is still valid here.

We know from the DICE model that a higher (lower) discount rate decreases (increases) the level of mitigation. In Fig. 6 we see that in the AD-DICE model a higher (lower) discount increases (decreases) the level of adaptation. This is quite intuitive as the benefits of mitigation are only felt in later periods, while the costs are felt in earlier periods. A higher (lower) discount rate would shift policy from mitigation (adaptation) to adaptation (mitigation).

\subsection{Climate sensitivity}

We do the same with three levels of climate sensitivity i.e. the equilibrium warming for a doubling of the atmospheric concentration of carbon dioxide. The original 


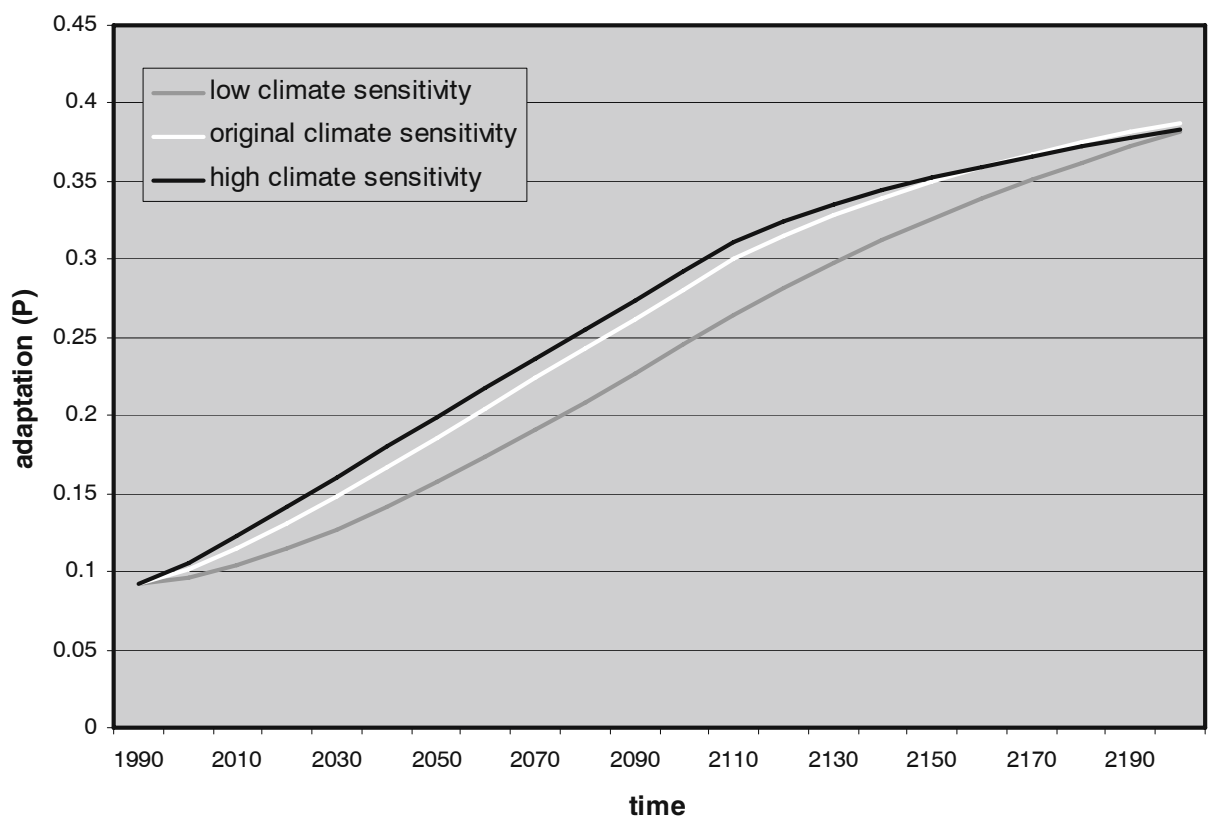

Fig. 7 Adaptation level with different climate sensitivities

climate-equation coefficient, that is the effect of radiative forcing on the atmospheric temperature, is 0.226 . This is equivalent to assuming a thermal capacity of the upper ocean layer of $0.0226 \frac{W y r}{K m^{2}}$ (cf. Füssel 2007). The low level coefficient is $50 \%$ of the original level (0.113) and the high level is $150 \%$ of the original level (0.339). Again we see that AD-DICE adjusts in the same manner as DICE with lower mitigation levels in later periods.

Lower (higher) climate sensitivity will decrease (increase) mitigation. In Fig. 7 we see that lower (higher) climate sensitivity will decrease (increase) adaptation until the year 2190 and increase (decrease) it after that. This is because if emissions cause less climate change, there will be lower damages. This will lead to lower levels of mitigation and adaptation. After some time due to the lower level of mitigation, damages will reach a level where a high level of adaptation will again become optimal.

The main point here is that our model replicates the DICE model with varying parameters without recalibration. That is, the analysis of mitigation policies in AD-DICE does not differ substantially from the analysis in DICE, even though the AD-DICE adaptation equations are only calibrated to one DICE scenario.

\subsection{Protection costs}

Finally we look at the results if we use another specification for the protection costs function. We give the $\mathrm{PC}_{t}$ function as

$$
\frac{\mathrm{PC}_{t}}{Y_{t}}=\gamma_{1}\left(\frac{1}{1-P_{t}}\right)^{\gamma_{2}}, \quad \text { where } \gamma_{1}>0 \text { and } \gamma_{2}>1 \text {. }
$$




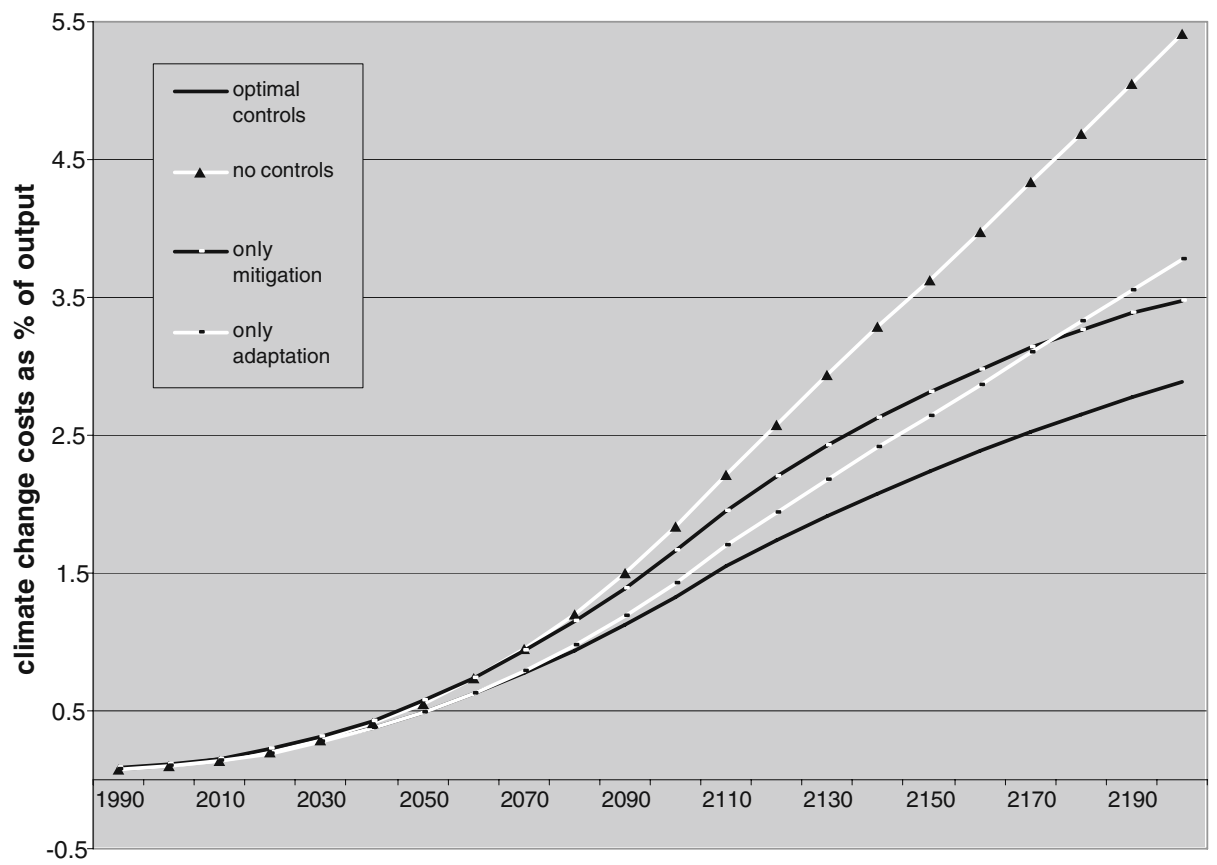

time

Fig. 8 Climate change costs of different scenarios with second $\mathrm{PC}_{t}$ specification

This full analysis is given in the Appendix 1; here we discuss the results only. The adaptation curves of both specifications are not identical, see Fig. 9 in Appendix 1. Using the alternative specification leads to a higher lying adaptation cost curve that translates into higher protection costs. The curve also becomes very steep at $P=0.3$ with extremely high costs at adaptation levels above that.

We again look at the policy scenarios with the new specification. The results show the same order of policies; however, the utility of the scenarios with no adaptation are slightly higher than before.

We also check to see whether the costs of climate change under the different control options develop in the same way as before and if the results are not sensitive to changes in specifications. In Fig. 8, we present the climate cost curves of the different options using the new protection cost function. We compare Fig. 8 with the corresponding results of the original protection cost function given in Fig. 4. The curves have the same form in both figures. Because the adaptation cost curve is slightly higher in this specification (see Fig. 9), the no control curve is slightly lower than before. We thus can conclude that our results on the policy options are robust to these changes.

Adaptation and mitigation are still affected by each other in the same way as in the original $\mathrm{PC}_{t}$ specification. Adaptation increases the benefits of mitigation in earlier periods and decreases them in later periods. Mitigation decreases the benefits of adaptation. This is not affected by our specification of the adaptation equations in AD-DICE. 


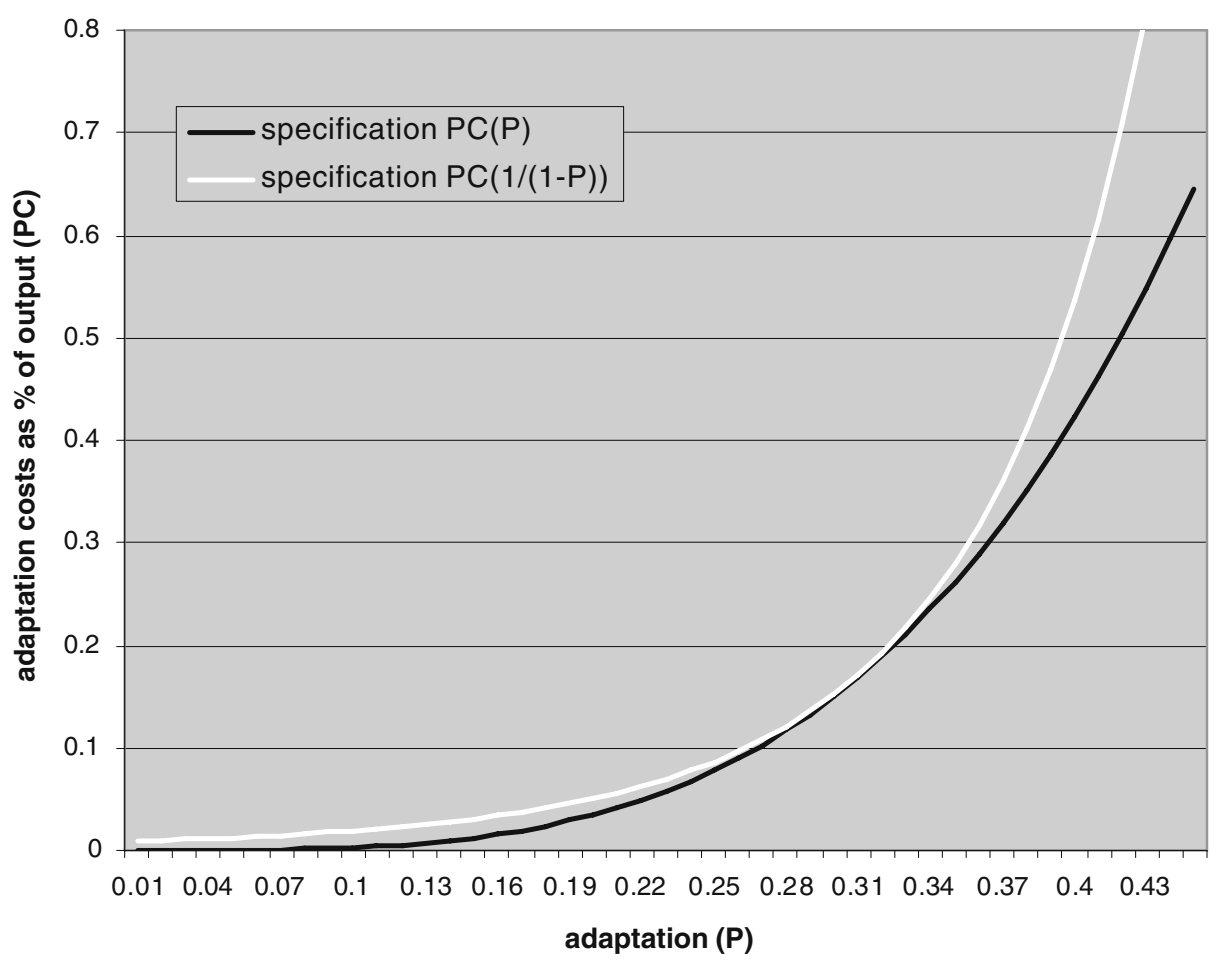

Fig. 9 Adaptation curves for both PC specifications

\section{Conclusions}

In this paper, we set up a framework that can model adaptation as a decision variable in IAMS that include a climate change damage module with monetised damages. We use this framework to create the model AD-DICE that includes adaptation as a decision variable in the DICE model of Nordhaus and Boyer (2000). In the DICE model, adaptation is assumed to be optimal and implicit in the damage function.

We adopt a framework in which adaptation and mitigation decisions are separable: the adaptation level is chosen to minimise net damages plus adaptation costs, while the mitigation level is chosen to minimise the aggregate of net damages and adaptation costs plus mitigation costs. AD-DICE is calibrated to mimic the original DICE model for the optimal control scenario and the best-guess parameters. Without recalibration, our model also replicates the mitigation results of the DICE model under different scenarios and parameter values. This means that making adaptation explicit and setting it at an optimal level yields the same results as implicit optimal adaptation. While this is obvious for the calibrated scenario, the fact that mitigation results in DICE are not affected by the implicit representation of adaptation implies that explicit treatment of adaptation does not alter the results concerning the economic impacts of mitigation policies. Thus we tentatively conclude that while adaptation may not be explicitly represented in most existing Integrated Assessment Models, there are no grounds to reject the mitigation policy conclusions from these 
models for the reason that they do not explicitly include adaptation. That is, while these models cannot be used to investigate optimal adaptation strategies, there are not necessarily biased in their analysis of mitigation strategies.

Our results show that both adaptation and mitigation can substantially reduce the impacts of climate change. When applied optimally, adaptation will reduce gross damages of climate change by on average $33 \%$ (as calibrated). We see that applying only adaptation is more beneficial than applying only mitigation, illustrating the importance of adaptation as a control option in combating climate change, especially in the short run. We also conclude that adaptation is the main climate change cost reducer in the first periods (up to the year 2100) after which mitigation reduces the bulk of the damages. Thus to combat climate change in the efficient way, the short term optimal policy consists of a mixture of adaptation measures and investments in mitigation, even though the latter will only decrease damages in later periods.

To challenge the conclusions from AD-DICE, more research is needed. Firstly, we ignore uncertainty. However, the way we calibrated the gross damage and adaptation functions makes them respond in the same way to key parameter variations as the original DICE model. This suggests that the behaviour of AD-DICE would be similar to that of DICE, also under uncertainty. Secondly, we ignore irreversibility. Should greenhouse gas emissions set in motion a shutdown of the thermohaline circulation or a collapse of the Greenland or West-Antarctic Ice Sheet, adaptation may be the only policy option. Thirdly, we assume perfect markets. We use an aggregate impact function, rather than making a distinction between different sectors. In some sectors, adaptation is relatively easy. In other sectors, it is more difficult. More detailed modelling of adaptation would also allow for a distinction between transition costs and equilibrium costs. Fourthly, we use a single region model. Like sectors, different countries may have a qualitatively different response to climate change, and adaptation may be very different. Furthermore, with different countries, countries may cooperate on mitigation, on adaptation, or both-while the original DICE model has only one instrument of international cooperation. Finally, adaptation may not be applied optimally but still applied to a certain extent. Therefore further research into non-optimal adaptation scenarios is warranted. All this is deferred to future work, which can build upon the framework developed in this paper.

\section{Appendix 1: an alternative specification of the adaptation cost function}

In this Appendix 1 we show the calibration of the AD-DICE model using another specification of the $\mathrm{PC}_{t}$ function. This specification is based on the idea that the $\mathrm{RD}_{t}$ function takes another form, i.e.

$$
\mathrm{RD}_{t}=\frac{\mathrm{GD}_{t}}{\mathrm{PR}_{t}}, \quad \text { where } \quad \mathrm{PR}_{t}>1
$$

Table 2 Parameter values from AD-DICE using the PC $(1 /(1-P))$ specification

\begin{tabular}{lllllll}
\hline Scenario & $\alpha_{1}$ & $\alpha_{2}$ & $\alpha_{3}$ & $\gamma_{1}$ & $\gamma_{2}$ & $p$-value \\
\hline Optimal & 0.00058 & 0.0027 & 2.20 & 0.000082 & 8.19 & 0.99 \\
\hline
\end{tabular}


The level of protection is given by $\mathrm{PR}_{t}$. Thus, instead of assuming that protection directly reduces gross damages on a one-to-one basis, we assume that adaptation becomes less effective as the level of adaptation rises. The costs of this protection we assume to be given by a power function:

$$
\frac{\mathrm{PC}_{t}}{Y_{t}}=\gamma_{1} \mathrm{PR}_{t}^{\gamma_{2}}, \quad \text { where } \quad \gamma_{1}>0 \quad \text { and } \quad \gamma_{2}>1
$$

To be able to use the logical interpretation of $P_{t}$ and to thus keep the original $\mathrm{RD}_{t}$ function (3), we rewrite Eq. 8 as a function of $P_{t}$, this results in the following $\mathrm{PC}_{t}$ function:

$$
\frac{\mathrm{PC}_{t}}{Y_{t}}=\gamma_{1}\left(\frac{1}{1-P_{t}}\right)^{\gamma_{2}}, \text { where } \quad \gamma_{1}>0 \text { and } \gamma_{2}>1
$$

The results of the calibration with this specification are given in Table 2. In Fig. 9 the adaptation curves under both specifications are given.

Open Access This article is distributed under the terms of the Creative Commons Attribution Noncommercial License which permits any noncommercial use, distribution, and reproduction in any medium, provided the original author(s) and source are credited.

\section{References}

de Bruin KC, Dellink RB, Agrawala S (forthcoming) Integrated assessment modelling of adaptation. OECD working paper

Dickinson T (2007) The compendium of adaptation models for climate change: first edition, adaptation and impacts research division, Environment Canada, $52 \mathrm{pp}$

Fankhauser S (1994) Protection vs. retreat-the economic costs of sea level rise. Environ Plan A 27:299-319

Fankhauser S (1998) The costs of adapting to climate change. GEF Working Paper Series 16, Global Environmental Facility, ISBN 1-884122-14-0

Füssel H-M (2007) Methodological and empirical flaws in the design and application of simple climate-economy models. Clim Change 81:161-185

Hope CW (2006) The marginal impact of $\mathrm{CO}_{2}$ from PAGE2002: an integrated assessment model incorporating the IPCC's five reasons for concern. Integr Assess J 6(1):19-56

Hope CW, Anderson J, Wenman P (1993) Policy analysis of the greenhouse effect-an application of the PAGE model. Energy Policy 15:328-338

IPCC (2001a) Climate change. In: Houghton JT et al (eds) Working group 1: the scientific basis. Cambridge University Press, Cambridge, UK

IPCC (2001b) Climate change. In: McCarthy JJ et al (eds) Working group II: impacts, adaptation and vulnerability. Cambridge University Press, Cambridge, UK

Klein RJT, Huq S, Denton F, Downing TE, Richels RG, Robinson JB, Toth FL (2007) Interrelationships between adaptation and mitigation. Climate Change 2007: impacts, adaptation and vulnerability. In: Parry ML, Canziani OF, Palutikof JP, van der Linden PJ, Hanson CE (eds) Contribution of working group II to the fourth assessment report of the intergovernmental panel on climate change. Cambridge University Press, Cambridge, UK, pp 745-777

Lecocq F, Shalizi Z (2007) Balancing expenditures on mitigation of and adaptation to climate change: an exploration of issues relevant to developing countries. World Bank Policy Research Working Paper No. 4299 Available at SSRN: http://ssrn.com/abstract=1004841

Manne AS, Mendelsohn R, Richels RG (1995) MERGE: a model for evaluating regional and global effects of GHG reduction policies. Energy Policy 23(1):17-34

Manne AS, Richels RG, Edmonds JA (2005) Market exchange rates or purchasing power parity: does the choice make a difference to the climate debate? Clim Change 71(1-2):1-8

Mendelsohn R (2000) Efficient adaptation to climate change. Clim Change 45:583-600 
Nordhaus WD (1994) Managing the global commons: the economics of the greenhouse effect. MIT Press, Cambridge, MA

Nordhaus WD, Boyer J (2000) Warming the world: economic models of global warming. MIT Press, Cambridge, MA

Parry ML, Carter TR, Konijn NT (eds) (1998a) The impact of climate variations on agriculture. Kluwer, Dordrecht

Parry ML, Arnell NW, Hulme M, Nicholls RJ, Livermore M (1998b) Adaptation to the inevitable. Nature 395:741

Plambeck EL, Hope CW, Anderson J (1997) The PAGE95 model: integrating the science and economics of climate change. Energy Econ 19:77-101

Reilly J, Hohnmann N, Kane S (1994) Climate change and agricultural trade: who benefits, who loses? Glob Environ Change 4(1):24-36

Smit B, Pilifosova O (2001) Adaptation to climate change in the context of sustainable development and equity. In: McCarthy JJ, Canziani O, Leary NA, Dokken DJ, White KS (eds) Climate change 2001: impacts, adaptation and vulnerability. Cambridge University Press, Cambridge, UK, pp 877-912

Sokolov AP, Schlosser CA, Dutkiewicz S, Paltsev S, Kicklighter DW, Jacoby HD, Prinn RG, Forest CE, Reilly JM, Wang C, Felzer BS, Sarofim MC, Scott J, Stone PH (2005) MIT integrated global system model (IGSM) version 2: model description and baseline evaluation, MIT Joint Program on the Science and Policy of Global Change, Report no. 124

Tol RSJ (2002) Estimates of the damage costs of climate change. Environ Resour Econ 21:47-73

Tol RSJ (2005) Adaptation and mitigation: trade-offs in substance and methods. Environ Sci Policy 8(6):572-578

Tol RSJ (2006) Exchange rates and climate change: an application of FUND. Clim Change 75:59-80

Tol RSJ (2007) The double trade-off between adaptation and mitigation for sea level rise: an application of FUND. Mitig Adapt Strategies Glob Change 12(5):741-753

Tol RSJ, Dowlatabadi H (2001) Vector-borne diseases, climate change, and economic growth. Integr Assess 2:173-181

Tol RSJ, Fankhauser S (1998) On the representation of impact in integrated assessment models of climate change. Environ Model Assess 3:63-74

Tol RSJ, Fankhauser S, Smith JB (1998) The scope for adaptation to climate change: what can we learn from the impact literature? Glob Environ Change 8(2):109-123 